\title{
Real-time online fingerprint image classification using adaptive hybrid techniques
}

\author{
Annapurna Mishra1, Satchidananda Dehuri ${ }^{2}$ \\ ${ }^{1}$ Department of Electronics and Communication Engineering, Silicon Institute of Technology, India \\ ${ }^{2}$ Department of Information and Communication Technology Fakir Mohan University, India
}

\section{Article Info \\ Article history: \\ Keywords: \\ $\mathrm{BBO}$ \\ Classification \\ Fingerprint \\ FLANN \\ Henry System}

ReceivedFeb 21, 2019

Revised Apr 18, 2019

Accepted Apr 30, 2019

\begin{abstract}
This paper presents three different hybrid classification techniques applied for the first time in real-time online fingerprint classification. Classification of online real time fingerprints is a complex task as it involves adaptation and tuning of classifier parameters for better classification accuracy. To accomplish the optimal adaptation of parameters of functional link artificial neural network (FLANN) for real-time online fingerprint classification, proven and established optimizers, such as Biogeography based optimizer (BBO), Genetic algorithm (GA), and Particle swarm optimizer (PSO) are intelligently infused with it to form hybrid classifiers. The global features of the real-time fingerprints are extracted using a Gabor filter-bank and then passed into adaptive hybrid classifiers for the desired classification as per the Henry system. Three hybrid classifiers, the optimized weight adapted Biogeography based optimized functional link artificial neural network (BBO-FLANN), Genetic algorithm based functional link artificial neural network (GA-FLANN) and Particle swarm optimized functional link artificial neural network (PSO-FLANN), are explored for real-time online fingerprint classification, where the PSO-FLANN technique is showing superior performance as compared to GA-FLANN and BBO-FLANN techniques. The best accuracy observed by the application of PSO-FLANN, is $98 \%$ for real-time online fingerprint classification.
\end{abstract}

Copyright (C) 2019Institute of Advanced Engineering and Science. All rights reserved.

\section{Corresponding Author:}

Annapurna Mishra,

Department of Electronics and Communication Engineering,

Silicon Institute of Technology, Silicon Hills,

Patia, Bhubaneswar-751024, Odisha, India.

Email: annapurnamishra12@gmail.com

\section{INTRODUCTION}

Fingerprint is the unique pattern of each and every individual human being and is the most widely used biometric authentication subject. For recognition as well as identification of fingerprints, it should be classified into different patterns called fingerprint class [1-2]. As per Henry system of fingerprints, it is divided into five types of unique patterns called Global class patterns [3], like two loop patterns (Left loop and Right loop), one whorl, and two arch patterns (Arch and Tented Arch) as given in Figure 1 [4]. The two arch patterns can be combined to be represented as one class. With this combination the total number of classes considered is 4 classes like Left loop, Right Loop, Whorl and Arch [5-6]. 


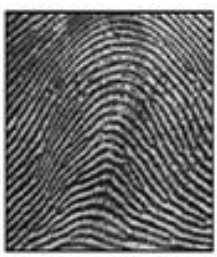

Arch

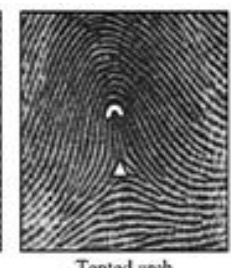

Tented anch

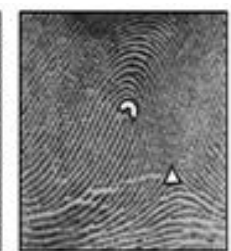

Left loop

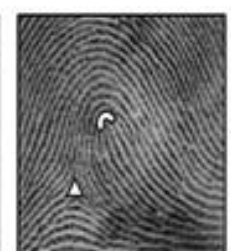

Right loop

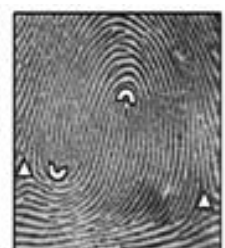

Whorl

Figure 1. Five fingerprint classes of henry system

For classification many researchers have developed efficient classifiers, and have tested on the available Fingerprint Database images like NIST, DB, and FVC etc. S.Dehuri et.al [7] has discussed regarding the classification accuracy of the combined efforts of FLANN and IPSO, which gives rise to a robust classifier interms of its architectural complexity as compared to MLP, SVM, RBF and FSN methods. B. Naik et.al [8] has explained the competence of HMBO algorithm for mating process and selection of best weights for FLANN classifiers. T. Dash et.al [9] has discussed regarding Fuzzy-MLP approach for non-linear pattern classification of fingerprints. A. K. Jain et.al [10] has discussed regarding the classification of fingerprint patterns into 5 classes and classified the fingerprints available in NIST-4 database. He achieved about $90 \%$ accuracy for five class classification and $94.8 \%$ accuracy for four class classification for offline database images. D. Simon [11] has discussed regarding the mathematics formulation of BBO and has seen that it has similar features like GA and PSO and can be applicable to similar optimization problems. The accuracy of the classification can be improved further with real-time database images using different hybrid classifiers.

In this work we have classified fingerprints of real time created database and here we basically focus on Hybrid classifiers, combining different optimizers along with FLANN classifier. First we have collected the results of classification algorithms like GA-FLANN, then BBO-FLANN and PSO-FLANN and then analyzed the results. After comparison, it is clear that PSO-FLANN is producing best results as compared to the other two algorithms

\section{PROPOSED METHOD}

\subsection{Introduction to FLANN as a classifier}

Here we have used the trigonometric expansion model, where each element of the input feature vector before expansion can be represented as, $r(i), 1<i<I$ where each element $\mathrm{r}(\mathrm{i})$ can be represented as $r_{n}(i), 1<n<N$, where $\mathrm{N}=$ number of expanded points for each input element [12][13]. In our case, $\mathrm{N}=11$ and $\mathrm{I}=$ represents the total number of features in the feature vector [14].The expansion can be represented as [15-16],

$$
x_{1}(i)=r(i), x_{2}(i)=\cos \pi(r(i)), x_{3}=\sin \pi(r(i)), \ldots \ldots \ldots \ldots x_{11}(i)=\sin 9 \pi(r(i))
$$

where, $r(i), 1<i<d, d$ is the set of features in the data set.

Then the random weights chosen from the range [11] are multiplied to the output and then added to produce the actual output of the network as given in Figure 2 [12]. For comparison the specified desired output is taken into consideration and the corresponding difference is the calculated error and is used to modify the weight in each path q, which can be expressed as [17],

$$
\Delta W_{j}(k)=\mu \times x f_{j}(k) \times e(k)
$$

where, $x f_{j}(k)$ is the functionally expanded input at $\mathrm{k}^{\text {th }}$ iteration.

For $q$ number of patterns, the change in weight is

$$
\overline{\Delta W_{j}(k)}=\frac{1}{q} \sum_{i=1}^{q} \Delta W_{j}^{i}(k)
$$


The weight updation is done by,

$$
W_{j}(k+1)=W_{j}(k)+\Delta W_{j}(k)
$$

Where, $W_{j}(k)$ is the $\mathrm{j}^{\text {th }}$ weight at the $\mathrm{k}^{\text {th }}$ iteration.

By taking $\mathrm{y}(\mathrm{k})$ as the desired output of the network, and $\hat{\mathrm{y}}(\mathrm{k})$ as the actual output of the network, the error $\mathrm{e}(\mathrm{k})$ can be calculated as,

$$
e(k)=y(k)-\hat{y}(k)
$$

Where $\hat{y}(k)=\sum_{j=1}^{J} x f_{j}(k) \cdot w_{j}(k)$

and $x f_{j}$ represents the expansion of input.

Though FLANN classifier is producing a very good accuracy of $100 \%$ for offline database, but for on-line real time classification the following drawbacks are present. The stabilizing factor $(\mu)$ needs to be tuned by the user by hit and trial method to achieve accuracy. Due to the manual tuning of the parameter, it fails to classify the real-time online fingerprint classification.

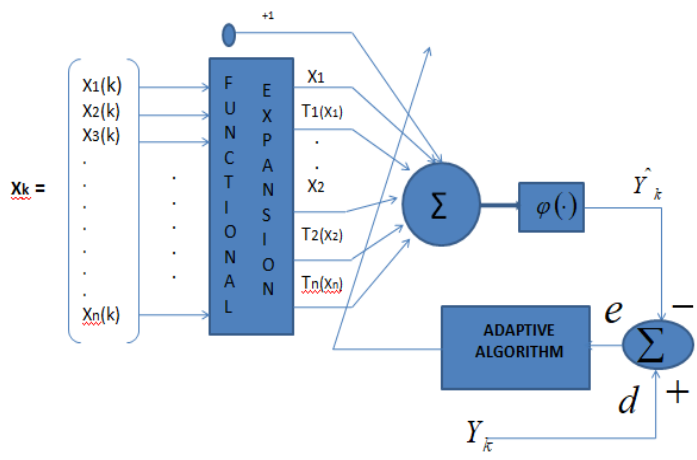

Figure 2. The structure of FLANN classifier

\subsection{Proposed adaptive hybrid classification techniques}

In this paper we are using three hybrid classifiers for real-time online classification and compared their results in terms of accuracy and execution time. The classifiers are:(i) BBO-FLANN, (ii) GA-FLANN, (iii) PSO-FLANN.

\subsubsection{BBO-FLANN}

This is a hybrid technique consisting of FLANN classifier along with Biogeography based optimization to optimize the different parameters of FLANN during online real-time processing of fingerprints. $\mathrm{BBO}$ has a property of estimating the parameters in geographical regions.

1. $\mathrm{BBO}$ as Optimized weight adapter

Biogeography Based Optimization basically focuses on species distribution within the neighbor islands [18-19], that means mathematically it is the description of species migration from island to island and development of new species. Here SIVs (Suitability index variables) indicate the habitability parameter, where as HSIs (Habitat Suitability Index) indicates the well suited residence for biological species, considering the features like weather condition, land area temperature etc [19]. In BBO, SIVs are independent variables of the habitat and HSI is the dependent variable. For optimization task, it follows the following steps:

a. Migration: Here the collection of candidate solutions that is population is represented in terms of vectors of integers or SIVs. The best fit solutions are taken as habitats with high HSI $\left(S_{2}\right)$ and least fit solutions are called low HSI $\left(S_{1}\right)$. The parameters $\lambda_{1}$ and $\mu_{1}$ represent the rate of immigration and emigration for $S_{1}$ and $\lambda_{2}$ and $\mu_{2}$ represent the rate of immigration and emigration for $S_{2}$ respectively. 
The $\lambda$ and $\mu$ values of each solution probabilistically share information between habitats. With a probability $P_{\text {mod }}$, each solution is further modified.

b. Mutation:The SIV mutation process involves the sudden change in habitat's HSI due to random events. The species count probabilities are used to determine mutation rates [18]. The mutation rate ' $m$ ' is inversely proportional to the solution probability.

2. BBO- FLANN technique for fingerprint classification

This work uses BBO as an optimizer to update the weight parameters of FLANN classifier. The steps are:Initially a fixed number of habitats are generated, where each habitat carries the respective weights and bias of the network. Then the best fit value in terms of MSE is calculated. Here the goal is to minimize the error with respect to the desired and the estimated output of the classifier. So to satisfy the optimization criteria, various operations like Initialization of habitat, migration, and mutation are performed and once the condition is satisfied it is terminated to find the best solution in terms of optimization. Then the network with high fitness (solution parameters) are passed to the next generation and repeated until the desired goal is achieved as given in Figure 3.

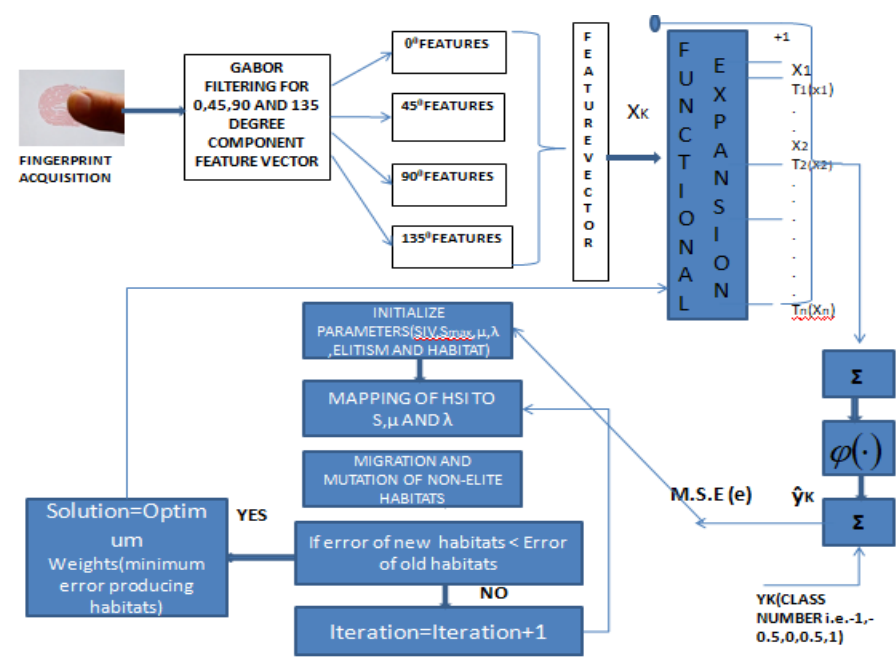

Figure 3. Structure of the proposed BBO-FLANN hybrid method

\subsubsection{GA-FLANN}

This is a hybrid technique consisting of FLANN classifier along with Genetic algorithm to optimize the different parameters of FLANN during online real-time processing of fingerprints. GA has a property of estimating the parameters with genetic programming through heuristic search.

\section{GA as Optimized weight adapter}

Genetic Algorithms (GAs) is a heuristic search process and an adaptive algorithm based on natural selection of genetics. It is an intelligent algorithm capable of solving optimization problem. It proves an intelligent random search to solve optimization problem [13, 20].GAs are designed to solve problems in natural systems those follow the principle of "survival of fittest". It is a robust search algorithm. Basically it follows the following steps:

a. Selection Operator: It is the first step in the search process which gives preference to better candidates and passes their respective genes for the next generation. The goodness criteria mostly depend on the fitness value and are decided by the objective function [21,22].

b. Crossover Operator: In this process two individual solutions are selected from the whole population. The string values of the individual solutions are exchanged by randomly selecting the point of crossover. This mating process creates two off-springs which are further used for the next generation $[13,22]$.

c. Mutation Operator: This process uses the low probability individual solutions where the bits are flipped for maintaining diversity. So mutation along with selection process combined together makes the algorithm robust and noise tolerant $[17,22]$. 
2. GA- FLANN technique for fingerprint classification

This work uses GA as an optimizer to update the weight parameters of FLANN classifier. The steps are:Initially a fixed number of populations are generated, where each population carries the respective weights and bias of the network. The best fit value as MSE is calculated. Here our intension is error minimization with respect to the desired and the estimated output of the classifier. So to satisfy the optimization criteria, various operations like Initialization of weights, elitism condition, Mutation and Crossover are performed and once the condition is satisfied it is terminated to find the best solution in terms of optimization.Then the network with high fitness (solution parameters) are passed to the next generation and repeated until the desired goal is achieved as given in Figure 4.

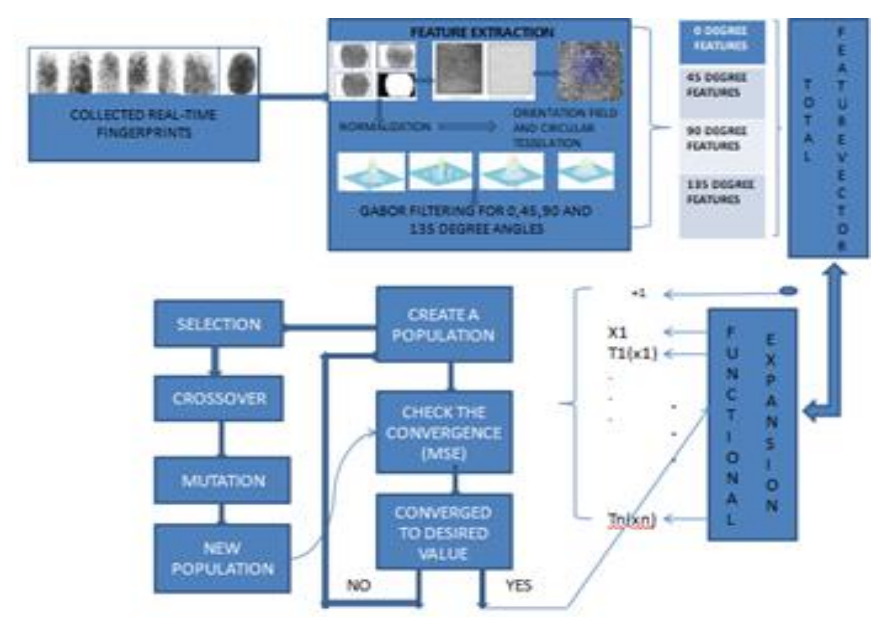

Figure 4. Structure of the proposed GA- FLANN hybrid method

\subsubsection{PSO-FLANN}

This is a hybrid technique consisting of FLANN classifier along with Particle Swarm optimization to optimize the different parameters of FLANN during online real-time processing of fingerprints. PSO has a property of estimating the parameters based on birds flocking or the natural phenomena.

\section{PSO as Optimized weight adapter}

Particle swarm optimization follows the population based algorithm that optimizes the objective function [12-13]. Here the solution is based on particles [13, 23], which imitates bird's flocking and are allowed to fly freely in the search space. In this process each and every particle are allowed to update their respective position and velocity for the whole population. PSO-FLANN technique for fingerprint classification.

This work uses PSO as an optimizer to update the weight parameters of FLANN classifier. The steps involved during this process are: initially a fixed number of habitats are generated, where each habitat carries the respective weights and bias of the network. The best fit value in terms of MSE is calculated. Here the goal is to minimize the error with respect to the desired and the estimated output of the classifier.To satisfy the optimization criteria, various operations like Initialization of weights, position and velocity update, memory update are performed and once the condition is satisfied it is terminated to find the best solution in terms of optimization. Then the network with high fitness (solution parameters) are passed to the next generation and repeated until the desired goal is achieved as given in Figure 5. 


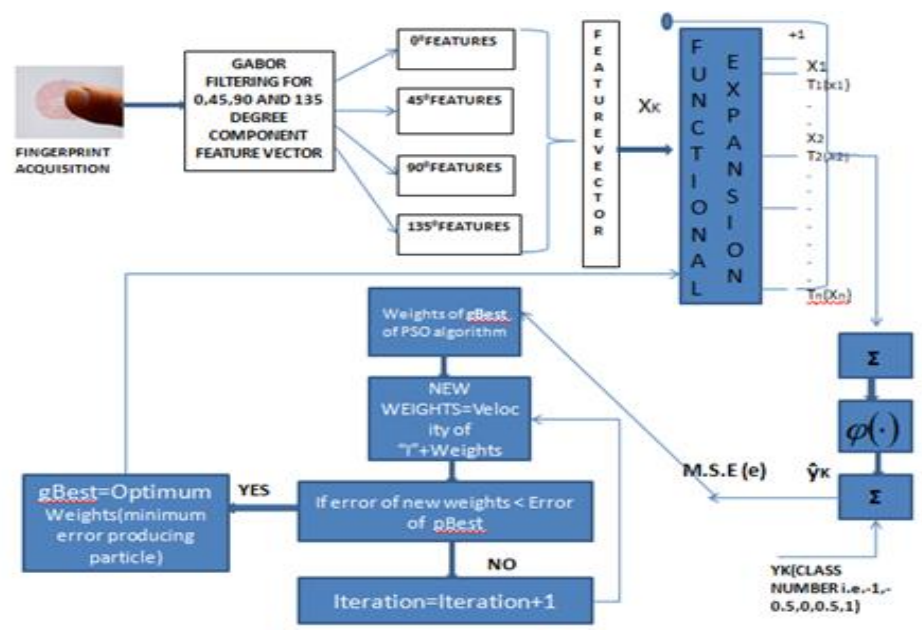

Figure 5. Structure of the proposed PSO- FLANN hybrid method

\section{RESEARCH METHOD}

\subsection{Generation of real-time database}

Classification of fingerprints is basically tested on standard database e.g. NIST 9.But in this work we have focused on generating a real time database of fingerprint samples. Here we have collected a group of 50 fingerprint sample images from 10 students of Silicon Institute of Technology, Bhubaneswar. The images are captured through fingerprint sensors and stored in a memory. So a total of 50 fingerprints consisting of all 4 classes of fingerprints originates the database for further processing task.

\subsection{Feature extraction and classification using proposed hybrid technique}

The groups of fingerprints are assigned to a particular class if the majority details are similar in both the samples. In order to assign a particular class to each one of the fingerprints, here the feature extraction step is done [24]. During feature extraction the following steps are carried out.

a. Feature vector creation

b. Normalization and Segmentation of fingerprint image

c. Orientation field estimation

d. Core point estimation

e. Circular region formation

f. Mean and Variance calculation for each of the sectors

g. Gabor filtering by 2-D convolution for 0, 45, 90, 135 degrees angles keeping a constant frequency separates the decision boundary to indicate the input class

\section{RESULTS AND DISCUSSION}

Selected degraded fingerprint images from the created and extracted real time database are feature extracted using filter bank approach and the feature excel sheet is created consisting of 152 features of each fingerprint image [14]. The extracted features of 50 real-time online fingerprint images in excel format are used for training and testing of the network. The sets of feature vectors are of all the classes starting from class 1 to class4.Here class4 (arch) and class5 (tented arch) jointly represent class4. Each class is represented in the excel sheet in terms of four values i.e. $(-1,-0.33,0.33,1)$ to represent the four classes respectively. It is tested for better performance for different iteration levels like 500, 1000, 2000, 3000, 5000 and 10,000 respectively. The test confusion matrix and the best cost graph are collected for analysis purpose.

From Table 1 it is clear that FLANN classifier is producing 100\% accuracy as shown in Figure 6, conidering the angle features and also for total feature vector in 64.16 seconds by taking the stabilizing factor $(\mu)$ with a value of 0.0005 . The stabilizing factor $(\mu)$ is a parameter which is tuned in hit and trail method in our classification process. So here the tuning of stabilizing factor $(\mu)$ is done randomly and manually to improve the accuracy which will create problem in real time on-line analysis.For selecting the stabilizing parameter in an adaptive process, the hybrid classifiers are used. 
Table 1. Classification accuracy and time duration of FLANN (for offline images)

\begin{tabular}{|c|c|c|c|c|c|}
\hline Sl. No. & $\begin{array}{c}\text { Stabilizing } \\
\text { Parameter }(\mu)\end{array}$ & Selected Feature Vector & Maximum Iteration & $\begin{array}{l}\text { Classification } \\
\text { Accuracy }(\%)\end{array}$ & $\begin{array}{l}\text { Time Duration } \\
\text { (in Seconds) }\end{array}$ \\
\hline \multirow{2}{*}{1} & \multirow{2}{*}{0.5} & \multirow{2}{*}{ Total feature vector } & 500 & 22 & 81.3 \\
\hline & & & 1000 & 26 & 176.08 \\
\hline \multirow{2}{*}{2} & \multirow{2}{*}{0.05} & \multirow{2}{*}{ Total feature vector } & 500 & 30 & 67.68 \\
\hline & & & 1000 & 30 & 133.48 \\
\hline \multirow{2}{*}{3} & \multirow{2}{*}{0.005} & \multirow{2}{*}{ Total feature vector } & 500 & 66 & 68.09 \\
\hline & & & 1000 & 62 & 133.11 \\
\hline \multirow{5}{*}{4} & \multirow{5}{*}{0.0005} & 0 degree & 500 & 100 & 21.12 \\
\hline & & 45 degree & 500 & 100 & 21.25 \\
\hline & & 90 degree & 500 & 100 & 21.34 \\
\hline & & 135 degree & 500 & 100 & 19.3 \\
\hline & & Total feature vector & 500 & 100 & 64.16 \\
\hline
\end{tabular}

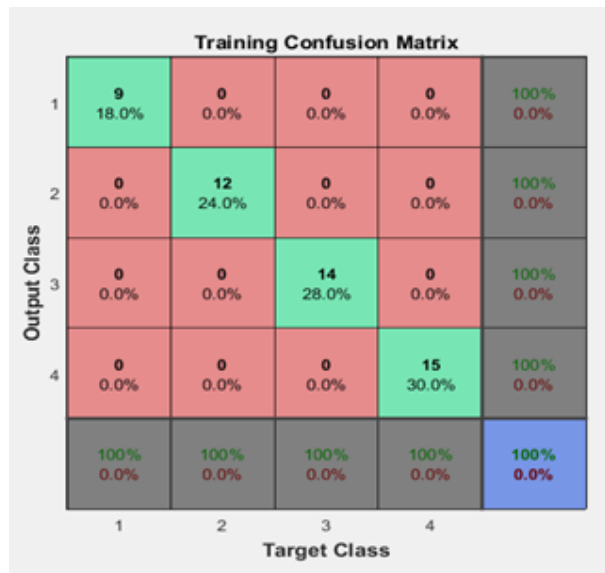

Figure 6. The Confusion matrix for total feature vector in FLANN (for offline database)

Table 2 shows the results of BBO-FLANN classification. It is producing an maximum accuracy of $82 \%$ for 90 degree feature vector with a bestcost value of 0.0727 in 891.79 seconds and $68 \%$ accuracy considering the total feature vector with a bestcost value of 0.13955 in $3.5657 \mathrm{e}+04$ seconds.Although this hybrid algorithm is producing good results of $82 \%$ for online fingerprints in real time platform, but the execution time is very high. Table 3 shows the results of GA-FLANN classification. It is producing an maximum accuracy of $100 \%$ for 90 degree feature vector with a bestcost value of 0.0087 in 143.9 seconds and $94 \%$ accuracy considering the total feature vector with a bestcost value of 0.09815 in $1.207 \mathrm{e}+03$ seconds. As per the result, the algorithm is robust enough for classification of different angular features as well as for total feature vector.

Table 2. Classification accuracy, best cost and time duration of BBO-FLANN (for online images)

\begin{tabular}{cccccc}
\hline S1. No. & $\begin{array}{c}\text { Selected Feature } \\
\text { Vector }\end{array}$ & $\begin{array}{c}\text { Maximum } \\
\text { Generation }\end{array}$ & $\begin{array}{c}\text { Best Cost } \\
\text { value }\end{array}$ & $\begin{array}{c}\text { Classification } \\
\text { Accuracy (\%) }\end{array}$ & $\begin{array}{c}\text { Time Duration } \\
\text { (in Seconds) }\end{array}$ \\
\hline 1 & 0 degree & 2000 & 0.11635 & 74 & 953.91 \\
2 & 45 degree & 10,000 & 0.08963 & 80 & $4.9397 \mathrm{e}+04$ \\
3 & 90 degree & 2000 & 0.0727 & 82 & 891.79 \\
4 & 135 degree & 10,000 & 0.10746 & 76 & $2.3923 \mathrm{e}+04$ \\
5 & Total feature vector & 10,000 & 0.13955 & 68 & $3.5657 \mathrm{e}+04$ \\
\hline
\end{tabular}

Table 3. Classification accuracy, best cost and time durationof GA-FLANN (for online images)

\begin{tabular}{cccccc}
\hline S1. No. & $\begin{array}{c}\text { Selected Feature } \\
\text { Vector }\end{array}$ & $\begin{array}{c}\text { Maximum } \\
\text { Generation }\end{array}$ & $\begin{array}{c}\text { Best Cost } \\
\text { value }\end{array}$ & $\begin{array}{c}\text { Classification } \\
\text { Accuracy (\%) }\end{array}$ & $\begin{array}{c}\text { Time Duration } \\
\text { (in Seconds) }\end{array}$ \\
\hline 1 & 0 degree & 10,000 & 0.10722 & 94 & 331.13 \\
2 & 45 degree & 20,000 & 0.18705 & 90 & 697.82 \\
3 & 90 degree & 5000 & 0.0087 & 100 & 143.9 \\
4 & 135 degree & 10,000 & 0.0269 & 94 & 228.96 \\
5 & Total feature vector & 10,000 & 0.09815 & 94 & $1.207 \mathrm{e}+03$ \\
\hline
\end{tabular}


Table 4 shows the results of PSO-FLANN classification. It is producing an maximum accuracy of $100 \%$ for 45 degree feature vector with a bestcost value of 0.017 in 73.47 seconds and $98 \%$ accuracy considering the total feature vector with a bestcost value of 0.022 in 146.65 seconds. As per the results, the algorithm is producing best results as compared to BBO-FLANN and GA-FLANN, and the stabilizing parameter is selected and optimized automatically, which makes it effective for real time online database. During the feature extraction stage a 152 dimensional feature vector is extracted by collectively considering four angle orientations $(0,45,90$ and 135 degrees) respectively. Here each angle orientation vector provides 38 no. of features and finally it forms 152 features for the whole fingerprint. The angular feature vector is tested in the network for $(0,45,90$ and 135 degrees) respectively and finally the whole feature vector is tested for accuracy. From the output graph as shown in Figure 7, it is clear that by providing the total feature vector as input to the network, it is providing best classification accuracy of $98 \%$ in PSO-FLANN algorithm where as it is $94 \%$ in GA-FLANN algorithm, which shows that PSO-FLANN is reflecting better results as compared to GA-FLANN. The best cost graphs and the confusion matrix are shown in the Figure 7.

Table 4. Classification accuracy, best cost and time duration of PSO-FLANN (for online images)

\begin{tabular}{cccccc}
\hline S1. No. & $\begin{array}{c}\text { Selected Feature } \\
\text { Vector }\end{array}$ & $\begin{array}{c}\text { Maximum } \\
\text { Generation }\end{array}$ & $\begin{array}{c}\text { Best Cost } \\
\text { value }\end{array}$ & $\begin{array}{c}\text { Classification } \\
\text { Accuracy }(\%)\end{array}$ & $\begin{array}{c}\text { Time Duration } \\
\text { (in Seconds) }\end{array}$ \\
\hline 1 & 0 degree & 500 & 0.015 & 98 & 85.64 \\
2 & 45 degree & 500 & 0.017 & 100 & 73.47 \\
3 & 90 degree & 500 & 0.036 & 92 & 71.9 \\
4 & 135 degree & 500 & 0.023 & 96 & 72.82 \\
5 & Total feature vector & 500 & 0.022 & 98 & 146.65 \\
\hline
\end{tabular}

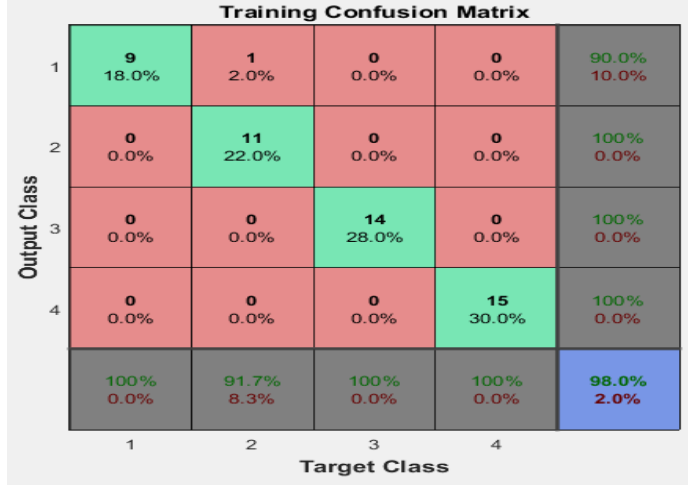

(a)

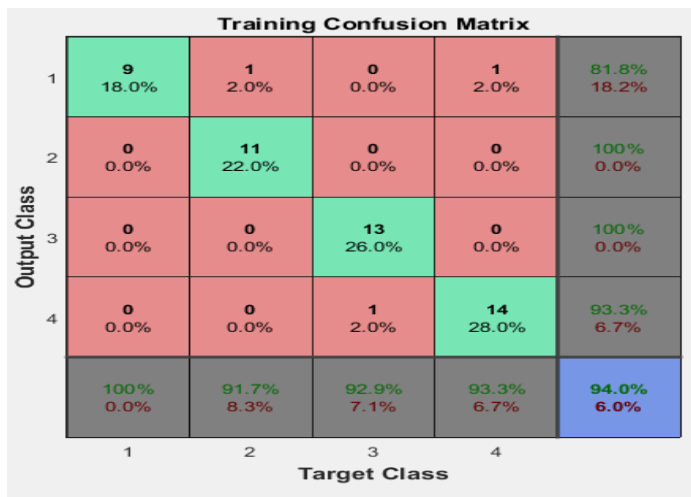

(c)

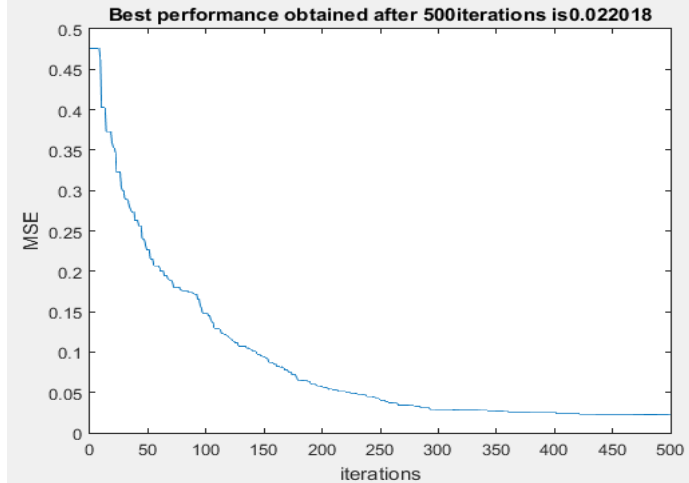

(b)

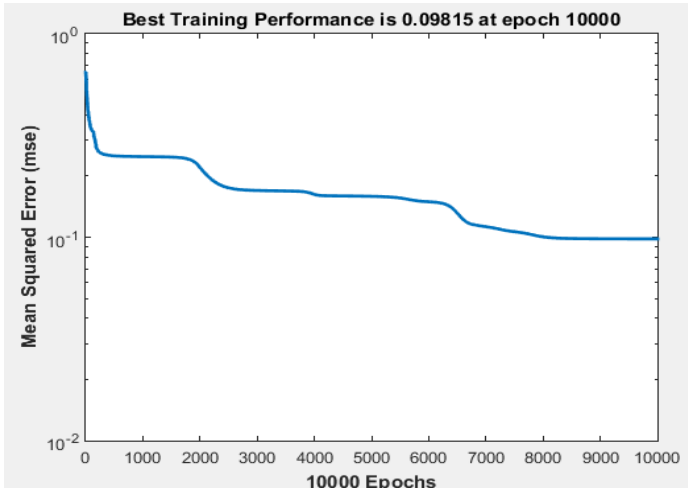

(d)

Figure 7. The Confusion matrix and best cost graph for total feature vector, (a) confusion matrix of PSOFLANN for total feature vector combining all angle feature vectors, (b) best cost graph of PSO-FLANN for total feature vector combining all angle feature vectors, (c) confusion matrix of GA-FLANN for total feature vector combining all angle feature vectors, (d) best cost graph of GA-FLANN for total feature vector combining all angle feature vectors 


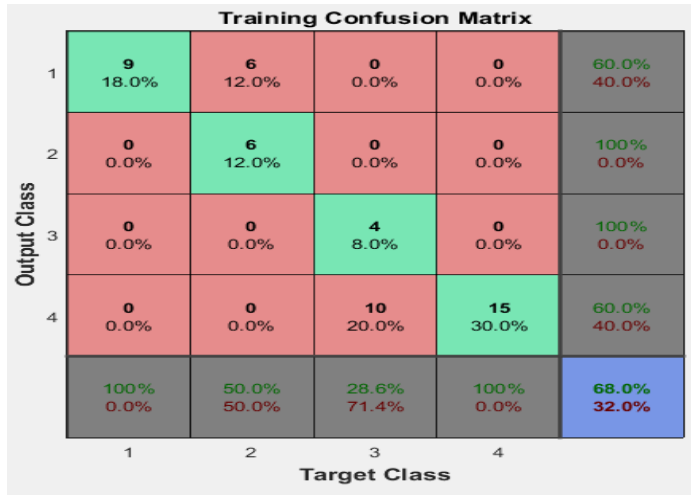

(e)

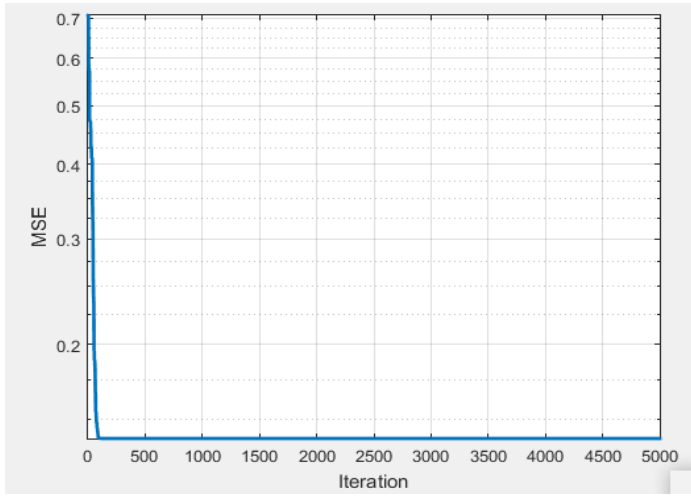

(f)

Figure 7. The Confusion matrix and best cost graph for total feature vector, (e) confusion matrix of BBOFLANN for total feature vector combining all angle feature vectors, (f) best cost graph of BBO-FLANN for total feature vector combining all angle feature vectors

\section{CONCLUSION}

In this paper, the classification of real-time fingerprints into four broad classes by adaptive hybrid classifiers is successfully carried out. During feature extraction the Gabor filter bank plays a major role for extracting the vital features of significance for the respective fingerprints. In the feature extraction stage a 152 dimensional feature vector is extracted for the whole fingerprint, by collectively considering four angles of orientations such as $0,45,90$ and 135 degrees, where each angle orientation vector provides 38 no. of features. The angular feature vectors for $0,45,90$ and 135 degrees as well as the total feature vector are used in the adaptive hybrid classifiers for testing classification accuracy.Here, unlike FLANN, where the tuning of stabilizing factor $(\mu)$ is done randomly and manually by hit and trial, to improve its classification accuracy, which is not possible in real time on line process, the adaptive hybrid classifiers like BBO-FLANN, GA-FLANN and PSO-FLANN are designed and tested for classification accuracy. As per the result, the PSO-FLANN technique is producing the maximum accuracy of $100 \%$ for 45 degree feature vector with a bestcost value of 0.017 and $98 \%$ accuracy considering the total feature vector with a bestcost value of 0.022 , which is the best classification accuracy as compared to BBO-FLANN and GA-FLANN. Here the stabilizing parameter $(\mu)$ is adapted and optimized automatically, which makes it effective for real time online fingerprint classification.

\section{ACKNOWLEDGEMENTS}

The first author would like to thank the technical support of Department of Information and Communication Technology, Fakir Mohan University, VyasaVihar, Balasore.

\section{REFERENCES}

[1] W. Bian, D. Xu, Q. Li, Y. Cheng, B. Jie and X. Ding, "A Survey of the Methods on Fingerprint Orientation Field Estimation," in IEEE Access, vol. 7, pp. 32644-32663, 2019.

[2] D. E. Hamdi, I. Elouedi, A. Fathallah, M. K. Nguyuen and A. Hamouda, "Combining Fingerprints and their Radon Transform as Input to Deep Learning for a Fingerprint Classification Task," 2018 15th International Conference on Control, Automation, Robotics and Vision (ICARCV), Singapore, pp. 1448-1453, 2018.

[3] Park, C.H. and Park, H., "Fingerprint classification using fast Fourier transform and nonlinear discriminant analysis," Pattern Recognition, pp.495-503, 2005.

[4] Li, Jun, Wei-Yun Yau, and Han Wang, "Combining singular points and orientation image information for fingerprint classification," Pattern Recognition 41, 353-366, 2008.

[5] Ratha, Nalini, and Ruud Bolle eds, "Automatic fingerprint recognition systems," Springer Science \& Business Media, 2003.

[6] Tan, X., Bhanu, B. and Lin, Y., "Fingerprint classification based on learned features," IEEE Transactions on Systems, Man, and Cybernetics, Part C (Applications and Reviews), pp.287-300, 2005.

[7] Dehuri, Satchidananda, Rahul Roy, Sung-Bae Cho, and Ashish Ghosh, "An improved swarm optimized functional link artificial neural network (ISO-FLANN) for classification," Journal of Systems and Software 85, no. 6, 1333-1345, 2012.

[8] Naik, Bighnaraj, Janmenjoy Nayak, and H. S. Behera, "A honey bee mating optimization based gradient descent learning-FLANN (HBMO-GDL-FLANN) for Classification," In Emerging ICT for Bridging the Future-Proceedings of the 49th Annual Convention of the Computer Society of India CSI Vol. 2, pp. 211-220, 2015. 
[9] Dash, Tirtharaj, Sanjib Kumar Nayak, and H. S. Behera, "Hybrid gravitational search and particle swarm based fuzzy MLP for medical data classification," In Computational Intelligence in Data Mining-Volume 1, pp. 35-43, Springer, New Delhi, 2015.

[10] Jain, Anil K., Salil Prabhakar, and Lin Hong, "A multichannel approach to fingerprint classification," IEEE transactions on pattern analysis and machine intelligence 21, no. 4, 348-359, 1999.

[11] Simon, D., "Biogeography-based optimization," IEEE transactions on evolutionary computation, pp.702-713, 2008.

[12] Dehuri, S., Roy, R., Cho, S.B. and Ghosh, A., "An improved swarm optimized functional link artificial neural network (ISO-FLANN) for classification,” Journal of Systems and Software, pp.1333-1345, 2012.

[13] Juang, C.F., "A hybrid of genetic algorithm and particle swarm optimization for recurrent network design," IEEE Transactions on Systems, Man, and Cybernetics, Part B (Cybernetics), pp.997-1006, 2004.

[14] Cho, M.Y. and Hoang, T.T., "Feature selection and parameters optimization of svm using particle swarm optimization for fault classification in power distribution systems," Computational intelligence and neuroscience, 2017.

[15] Patra, J.C., Pal, R.N., Chatterji, B.N. and Panda, G., "Identification of nonlinear dynamic systems using functional link artificial neural networks," IEEE transactions on systems, man, and cybernetics, part b (cybernetics), pp.254-262, 1999.

[16] Kumar, P.S. and Valarmathy, S., "March. Development of a novel algorithm for SVMBDT fingerprint classifier based on clustering approach," In IEEE-International Conference On Advances In Engineering, Science And Management (ICAESM-2012), pp. 256-261, 2012.

[17] Javed, S. and Usman, A., "Computerized system for fingerprint classification using singular points," In 2011 IEEE 14th International Multitopic Conference, pp. 96-101, December 2011.

[18] Bhattacharya, A. and Chattopadhyay, P.K., "Hybrid differential evolution with biogeography-based optimization for solution of economic load dispatch," IEEE transactions on power systems, pp.1955-1964, 2010.

[19] Dehuri, S., Mishra, B.B. and Cho, S.B., "Genetic feature selection for optimal functional link artificial neural network in classification," In International Conference on Intelligent Data Engineering and Automated Learning, pp. 156-163. Springer, Berlin, Heidelberg, November, 2008.

[20] Siriwardene, N.R. and Perera, B.J.C., "Selection of genetic algorithm operators for urban drainage model parameter optimisation," Mathematical and Computer Modelling, 44(5-6), pp.415-429, 2006.

[21] Johnson, J.M. and Rahmat-Samii, Y., 1994, "Genetic algorithm optimization and its application to antenna design," In Proceedings of IEEE Antennas and Propagation Society International Symposium and URSI National Radio Science Meeting, Vol. 1, pp. 326-329, IEEE., June 1994.

[22] Simon, D., "Biogeography-based optimization," IEEE transactions on evolutionary computation, pp.702-713, 2008.

[23] Guo, Y.H. and Huang, C.L., "Functional link artificial neural networks filter for Gaussian noise," In Applied Mechanics and Materials, Vol. 347, pp. 2580-2585, 2013.

[24] Jain, A.K., Prabhakar, S. and Hong, L., "A multichannel approach to fingerprint classification," IEEE transactions on pattern analysis and machine intelligence, pp. 348-359, 1999. 